\title{
AN INVARIANT THEORY OF MARGINALLY TRAPPED SURFACES IN THE FOUR-DIMENSIONAL MINKOWSKI SPACE
}

\author{
GEORGI GANCHEV AND VELICHKA MILOUSHEVA
}

\begin{abstract}
A marginally trapped surface in the four-dimensional Minkowski space is a spacelike surface whose mean curvature vector is lightlike at each point. We associate a geometrically determined moving frame field to such a surface and using the derivative formulas for this frame field we obtain seven invariant functions. Our main theorem states that these seven invariants determine the surface up to a motion in Minkowski space.

We introduce meridian surfaces as one-parameter systems of meridians of a rotational hypersurface in the four-dimensional Minkowski space. We find all marginally trapped meridian surfaces.
\end{abstract}

\section{INTRODUCTION}

The concept of trapped surfaces was introduced by Roger Penrose in [14] and it plays an important role in general relativity. A surface in a 4-dimensional spacetime is called marginally trapped if it is closed, embedded, spacelike and its mean curvature vector is lightlike at each point of the surface. These surfaces were defined by Penrose in order to study global properties of spacetime. In Physics similar or weaker definitions attract attention. Recently, marginally trapped surfaces have been studied from a mathematical viewpoint. In the mathematical literature, it is customary to call a codimension-two surface in a 4-dimensional semi-Riemannian manifold marginally trapped it its mean curvature vector $H$ is lightlike at each point, and removing the other hypotheses, i.e. the surface does not need to be closed or embedded. Classification results in 4-dimensional Lorentz manifolds were obtained imposing some extra conditions on the mean curvature vector, the Gauss curvature or the second fundamental form. Marginally trapped surfaces with positive relative nullity in Lorenz space forms were classified in [2. The non-existence of marginally trapped surfaces in Robertson-Walker spaces with positive relative nullity was shown in [3]. Marginally trapped surfaces with parallel mean curvature vector in Lorenz space forms were classified in [4]. In [11 marginally trapped surfaces which are invariant under a boost transformation in 4-dimensional Minkowski space were studied, and marginally trapped surfaces in Minkowski 4-space which are invariant under spacelike rotations were classified in [12]. The classification of marginally trapped surfaces in Minkowski 4-space which are invariant under a group of screw rotations (a group of Lorenz rotations with an invariant lightlike direction) is obtained in [13].

In this paper, we consider marginally trapped surfaces in the four-dimensional Minkowski space $\mathbb{R}_{1}^{4}$. Our study is based on the geometrically introduced invariant linear map of Weingartentype in the tangent plane at any point of the surface under consideration. This allows us to introduce principal lines and geometrically determined invariant moving frame field. Writing derivative formulas of Frenet-type for this frame field, we obtain seven invariant functions and prove a fundamental theorem of Bonnet-type, stating that these seven invariants under some natural conditions determine the surface up to a motion in $\mathbb{R}_{1}^{4}$.

2000 Mathematics Subject Classification. Primary 53A35, Secondary 53B25.

Key words and phrases. Marginally trapped surfaces in the four-dimensional Minkowski space, lightlike mean curvature vector, Bonnet-type fundamental theorem, meridian surfaces in Minkowski space. 
We apply our theory to spacelike surfaces lying on rotational hypersurfaces in $\mathbb{R}_{1}^{4}$. Considering rotational hypersurfaces with timelike or spacelike axis we construct special two-dimensional surfaces which are one-parameter systems of meridians of the rotational hypersurface (meridian surfaces). We find all meridian surfaces which are marginally trapped.

\section{Preliminaries}

In [8] we considered the local theory of spacelike surfaces in the four-dimensional Minkowski space $\mathbb{R}_{1}^{4}$. The basic feature of our treatment of these surfaces was the introduction of an invariant linear map of Weingarten-type in the tangent plane at any point of the surface, following the approach to the theory of surfaces in $\mathbb{R}^{4}$ [5, 7]. Studying surfaces in the Euclidean space $\mathbb{R}^{4}$, in [5] we introduced a linear map $\gamma$ of Weingarten-type, which plays a similar role in the theory of surfaces in $\mathbb{R}^{4}$ as the Weingarten map in the theory of surfaces in $\mathbb{R}^{3}$. The map $\gamma$ generates the corresponding second fundamental form $I I$ at any point of the surface in the standard way. We gave a geometric interpretation of the second fundamental form and the Weingarten map of the surface in [6].

Let $\mathbb{R}_{1}^{4}$ be the Minkowski space endowed with the metric $\langle$,$\rangle of signature (3,1)$ and $O e_{1} e_{2} e_{3} e_{4}$ be a fixed orthonormal coordinate system in $\mathbb{R}_{1}^{4}$, i.e. $e_{1}^{2}=e_{2}^{2}=e_{3}^{2}=1, e_{4}^{2}=-1$, giving the orientation of $\mathbb{R}_{1}^{4}$. The standard flat metric is given in local coordinates by $d x_{1}^{2}+d x_{2}^{2}+d x_{3}^{2}-d x_{4}^{2}$.

A surface $M^{2}$ in $\mathbb{R}_{1}^{4}$ is said to be spacelike if $\langle$,$\rangle induces a Riemannian metric g$ on $M^{2}$. Thus at each point $p$ of a spacelike surface $M^{2}$ we have the following decomposition

$$
\mathbb{R}_{1}^{4}=T_{p} M^{2} \oplus N_{p} M^{2}
$$

with the property that the restriction of the metric $\langle$,$\rangle onto the tangent space T_{p} M^{2}$ is of signature $(2,0)$, and the restriction of the metric $\langle$,$\rangle onto the normal space N_{p} M^{2}$ is of signature $(1,1)$.

Denote by $\nabla^{\prime}$ and $\nabla$ the Levi Civita connections on $\mathbb{R}_{1}^{4}$ and $M^{2}$, respectively. Let $x$ and $y$ denote vector fields tangent to $M$ and let $\xi$ be a normal vector field. Then the formulas of Gauss and Weingarten give a decomposition of the vector fields $\nabla_{x}^{\prime} y$ and $\nabla_{x}^{\prime} \xi$ into a tangent and a normal component:

$$
\begin{aligned}
& \nabla_{x}^{\prime} y=\nabla_{x} y+\sigma(x, y) \\
& \nabla_{x}^{\prime} \xi=-A_{\xi} x+D_{x} \xi
\end{aligned}
$$

which define the second fundamental tensor $\sigma$, the normal connection $D$ and the shape operator $A_{\xi}$ with respect to $\xi$. The mean curvature vector field $H$ of the surface $M^{2}$ is defined as $H=\frac{1}{2} \operatorname{tr} \sigma$, i.e. given a local orthonormal frame $\{x, y\}$ of the tangent bundle, $H=\frac{1}{2}(\sigma(x, x)+\sigma(y, y))$.

Let $M^{2}: z=z(u, v),(u, v) \in \mathcal{D}\left(\mathcal{D} \subset \mathbb{R}^{2}\right)$ be a local parametrization on a spacelike surface in $\mathbb{R}_{1}^{4}$. The tangent space at an arbitrary point $p=z(u, v)$ of $M^{2}$ is $T_{p} M^{2}=\operatorname{span}\left\{z_{u}, z_{v}\right\}$. Since $M^{2}$ is spacelike, $\left\langle z_{u}, z_{u}\right\rangle>0,\left\langle z_{v}, z_{v}\right\rangle>0$. We use the standard denotations $E(u, v)=$ $\left\langle z_{u}, z_{u}\right\rangle, F(u, v)=\left\langle z_{u}, z_{v}\right\rangle, G(u, v)=\left\langle z_{v}, z_{v}\right\rangle$ for the coefficients of the first fundamental form

$$
I(\lambda, \mu)=E \lambda^{2}+2 F \lambda \mu+G \mu^{2}, \lambda, \mu \in \mathbb{R} .
$$

Since $I(\lambda, \mu)$ is positive definite we set $W=\sqrt{E G-F^{2}}$. We choose a normal frame field $\left\{n_{1}, n_{2}\right\}$ such that $\left\langle n_{1}, n_{1}\right\rangle=1,\left\langle n_{2}, n_{2}\right\rangle=-1$, and the quadruple $\left\{z_{u}, z_{v}, n_{1}, n_{2}\right\}$ is positively 
oriented in $\mathbb{R}_{1}^{4}$. Then we have the following derivative formulas:

$$
\begin{aligned}
\nabla_{z_{u}}^{\prime} z_{u} & =z_{u u}=\Gamma_{11}^{1} z_{u}+\Gamma_{11}^{2} z_{v}+c_{11}^{1} n_{1}-c_{11}^{2} n_{2} \\
\nabla_{z_{u}}^{\prime} z_{v} & =z_{u v}=\Gamma_{12}^{1} z_{u}+\Gamma_{12}^{2} z_{v}+c_{12}^{1} n_{1}-c_{12}^{2} n_{2} \\
\nabla_{z_{v}}^{\prime} z_{v} & =z_{v v}=\Gamma_{22}^{1} z_{u}+\Gamma_{22}^{2} z_{v}+c_{22}^{1} n_{1}-c_{22}^{2} n_{2}
\end{aligned}
$$

where $\Gamma_{i j}^{k}$ are the Christoffel's symbols and the functions $c_{i j}^{k}, i, j, k=1,2$ are given by

$$
\begin{array}{lll}
c_{11}^{1}=\left\langle z_{u u}, n_{1}\right\rangle ; & c_{12}^{1}=\left\langle z_{u v}, n_{1}\right\rangle ; & c_{22}^{1}=\left\langle z_{v v}, n_{1}\right\rangle ; \\
c_{11}^{2}=\left\langle z_{u u}, n_{2}\right\rangle ; & c_{12}^{2}=\left\langle z_{u v}, n_{2}\right\rangle ; & c_{22}^{2}=\left\langle z_{v v}, n_{2}\right\rangle .
\end{array}
$$

Obviously, the surface $M^{2}$ lies in a 2-plane if and only if $M^{2}$ is totally geodesic, i.e. $c_{i j}^{k}=$ $0, i, j, k=1,2$. So, we assume that at least one of the coefficients $c_{i j}^{k}$ is not zero.

Considering the tangent space $T_{p} M^{2}$ at a point $p \in M^{2}$, in [8] we introduced an invariant $\zeta_{g_{1}, g_{2}}$ of a pair of two tangents $g_{1}, g_{2}$ using the second fundamental tensor $\sigma$ of $M^{2}$. By means of this invariant we defined conjugate, asymptotic, and principal tangents. The second fundamental form $I I$ of the surface $M^{2}$ at a point $p \in M^{2}$ is introduced on the base of conjugacy of two tangents at the point. The coefficients $L, M, N$ of the second fundamental form $I I$ are determined as follows:

$$
L=\frac{2}{W}\left|\begin{array}{cc}
c_{11}^{1} & c_{12}^{1} \\
c_{11}^{2} & c_{12}^{2}
\end{array}\right| ; \quad M=\frac{1}{W}\left|\begin{array}{cc}
c_{11}^{1} & c_{22}^{1} \\
c_{11}^{2} & c_{22}^{2}
\end{array}\right| ; \quad N=\frac{2}{W}\left|\begin{array}{cc}
c_{12}^{1} & c_{22}^{1} \\
c_{12}^{2} & c_{22}^{2}
\end{array}\right| .
$$

The second fundamental form $I I$ determines an invariant linear map $\gamma$ of Weingarten-type at any point of the surface, which generates two invariant functions:

$$
k:=\operatorname{det} \gamma=\frac{L N-M^{2}}{E G-F^{2}}, \quad \varkappa:=-\frac{1}{2} \operatorname{tr} \gamma=\frac{E N+G L-2 F M}{2\left(E G-F^{2}\right)} .
$$

The functions $k$ and $\varkappa$ are invariant under changes of the parameters of the surface and changes of the normal frame field. The sign of $k$ is invariant under congruences and the sign of $\varkappa$ is invariant under motions in $\mathbb{R}_{1}^{4}$. However, the sign of $\varkappa$ changes under symmetries with respect to a hyperplane in $\mathbb{R}_{1}^{4}$. We proved that the invariant $\varkappa$ is the curvature of the normal connection of the surface. The number of asymptotic tangents at a point of $M^{2}$ is determined by the sign of the invariant $k$. In the case $k=0$ there exists a one-parameter family of asymptotic lines, which are principal.

It is interesting to note that the "umbilical" points, i.e. points at which the coefficients of the first and the second fundamental forms are proportional, are exactly the points at which the mean curvature vector $H$ is zero. So, the spacelike surfaces consisting of "umbilical" points in $\mathbb{R}_{1}^{4}$ are exactly the minimal surfaces. Minimal spacelike surfaces are characterized in terms of the invariants $k$ and $\varkappa$ by the equality $\varkappa^{2}-k=0$.

Analogously to $\mathbb{R}^{3}$ and $\mathbb{R}^{4}$, the invariants $k$ and $\varkappa$ divide the points of $M^{2}$ into four types: flat, elliptic, hyperbolic and parabolic points. The surfaces consisting of flat points are characterized by the conditions $k=\varkappa=0$, or equivalently $L=M=N=0$. We gave a local geometric description of spacelike surfaces consisting of flat points whose mean curvature vector at any point is a non-zero spacelike vector or timelike vector, proving that any such a surface either lies in a hyperplane of $\mathbb{R}_{1}^{4}$ or is part of a developable ruled surface in $\mathbb{R}_{1}^{4}[8]$.

Using the introduced principal lines on a spacelike surface in $\mathbb{R}_{1}^{4}$ whose mean curvature vector at any point is a non-zero spacelike vector or timelike vector, we found a geometrically determined moving frame field on such a surface. Writing the derivative formulas of Frenet-type for this frame field, we obtained eight invariant functions and proved a fundamental theorem of 
Bonnet-type, stating that these eight invariants under some natural conditions determine the surface up to a motion in $\mathbb{R}_{1}^{4}$.

In the present paper we shall apply the same idea for developing the invariant theory of spacelike surfaces in $\mathbb{R}_{1}^{4}$ whose mean curvature vector at any point is a lightlike vector, i.e. marginally trapped surfaces.

\section{INVARIANTS OF A MARGINALLY TRAPPED SURFACE}

Let $M^{2}: z=z(u, v),(u, v) \in \mathcal{D}$ be a marginally trapped surface. Then the mean curvature vector is lightlike at each point of the surface, i.e. $\langle H, H\rangle=0$. Thus there exists a pseudoorthonormal normal frame field $\left\{n_{1}, n_{2}\right\}$, such that $n_{1}=H$ and

$$
\left\langle n_{1}, n_{1}\right\rangle=0 ; \quad\left\langle n_{2}, n_{2}\right\rangle=0 ; \quad\left\langle n_{1}, n_{2}\right\rangle=-1 .
$$

We assume that $M^{2}$ is free of flat points, i.e. $(L, M, N) \neq(0,0,0)$. Then at each point of the surface there exist principal lines and without loss of generality we assume that $M^{2}$ is parameterized by principal lines. Let us denote $x=\frac{z_{u}}{\sqrt{E}}, y=\frac{z_{v}}{\sqrt{G}}$. Then $\sigma(x, x)$ and $\sigma(y, y)$ are collinear with the mean curvature vector field. So, we have the following decompositions

$$
\begin{aligned}
& \sigma(x, x)=(1+\nu) n_{1} ; \\
& \sigma(x, y)=\lambda n_{1}+\mu n_{2} ; \\
& \sigma(y, y)=(1-\nu) n_{1},
\end{aligned}
$$

where $\nu, \lambda, \mu$ are invariant functions, determined by the principal directions $x, y$, and the mean curvature vector field $n_{1}=H$ as follows:

$$
\nu=-\left\langle\frac{\sigma(x, x)-\sigma(y, y)}{2}, n_{2}\right\rangle, \quad \lambda=-\left\langle\sigma(x, y), n_{2}\right\rangle, \quad \mu=-\left\langle\sigma(x, y), n_{1}\right\rangle
$$

Using (11) and (2) we calculate the coefficients $L, M, N$ of the second fundamental form and find the invariants $k, \varkappa$ and the Gauss curvature $K$ of $M^{2}$ expressed by the functions $\nu, \lambda$, and $\mu$ :

$$
k=4 \mu^{2}\left(\nu^{2}-1\right) ; \quad \varkappa=-2 \mu \nu ; \quad K=2 \lambda \mu .
$$

Since $H \neq 0$, we have $\varkappa^{2}-k>0$. From (3) we get the relations:

$$
4 \mu^{2}=\varkappa^{2}-k ; \quad \lambda=\frac{K}{\sqrt{\varkappa^{2}-k}}, \quad \nu=-\frac{\varkappa}{\sqrt{\varkappa^{2}-k}} .
$$

The last equalities show the geometric meaning of the invariants $\nu, \lambda$, and $\mu$. Note that $\mu \neq 0$, since $\varkappa^{2}-k>0$.

Recall that a surface is called flat if the Gauss curvature $K$ vanishes; $M^{2}$ is with flat normal connection if and only if $\varkappa=0 ; M^{2}$ consists of parabolic points if and only if $k=0$. So, equalities (3) imply the following statements.

Proposition 3.1. Let $M^{2}$ be a marginally trapped surface free of flat points. Then $M^{2}$ is a flat surface if and only if $\lambda=0$.

Proposition 3.2. Let $M^{2}$ be a marginally trapped surface free of flat points. Then $M^{2}$ is a surface with flat normal connection if and only if $\nu=0$.

Proposition 3.3. Let $M^{2}$ be a marginally trapped surface free of flat points. Then $M^{2}$ is a surface consisting of parabolic points if and only if $\nu= \pm 1$. 
The flat marginally trapped surfaces can also be characterized in terms of the so called null allied mean curvature vector field. The allied vector field of a normal vector field $\xi$ of an $n$ dimensional submanifold $M^{n}$ of $(n+m)$-dimensional Riemannian manifold $\widetilde{M}^{n+m}$ is defined by B.-Y. Chen [1] by the formula

$$
a(\xi)=\frac{\|\xi\|}{n} \sum_{k=2}^{m}\left\{\operatorname{tr}\left(A_{1} \circ A_{k}\right)\right\} \xi_{k},
$$

where $\left\{\xi_{1}=\frac{\xi}{\|\xi\|}, \xi_{2}, \ldots, \xi_{m}\right\}$ is a local orthonormal frame of the normal bundle of $M^{n}$, and $A_{i}=A_{\xi_{i}}, i=1, \ldots, m$ is the shape operator with respect to $\xi_{i}$. In particular, the allied vector field $a(H)$ of the mean curvature vector field $H$ is a well-defined normal vector field which is called the allied mean curvature vector field of $M^{n}$ in $\widetilde{M}^{n+m}$. B.-Y. Chen defined the $\mathcal{A}$-submanifolds to be those submanifolds of $\widetilde{M^{n+m}}$ for which $a(H)$ vanishes identically [1]. In [9, 10] the $\mathcal{A}$-submanifolds are called Chen submanifolds. It is easy to see that minimal submanifolds, pseudo-umbilical submanifolds and hypersurfaces are Chen submanifolds. These Chen submanifolds are said to be trivial $\mathcal{A}$-submanifolds.

In [13] S. Haesen and M. Ortega extended the notion of allied mean curvature vector field to the case when the normal space is a two-dimensional Lorenz space and the mean curvature vector field is lightlike as follows. Denote by $\left\{H, H^{\perp}\right\}$ a pseudo-orthonormal basis of the normal space such that $\langle H, H\rangle=0 ;\left\langle H^{\perp}, H^{\perp}\right\rangle=0 ;\left\langle H, H^{\perp}\right\rangle=-1$. The null allied mean curvature vector field is defined as

$$
a(H)=\frac{1}{2} \operatorname{tr}\left(A_{H} \circ A_{H^{\perp}}\right) H^{\perp} .
$$

Now, if $M^{2}$ is a marginally trapped surface, then using equalities (2) we get

$$
A_{H}=A_{n_{1}}=\left(\begin{array}{cc}
0 & -\mu \\
-\mu & 0
\end{array}\right) ; \quad A_{H^{\perp}}=A_{n_{2}}=\left(\begin{array}{cc}
-(1+\nu) & -\lambda \\
-\lambda & -(1-\nu)
\end{array}\right) .
$$

Applying formula (4) and using (3), we obtain that the null allied mean curvature vector field of $M^{2}$ is expressed as follows:

$$
a(H)=\lambda \mu n_{2}=\frac{K}{2} n_{2} .
$$

Thus we obtain the following result.

Proposition 3.4. Let $M^{2}$ be a marginally trapped surface free of flat points. Then $M^{2}$ is a flat surface if and only if $M^{2}$ has vanishing null allied mean curvature vector field.

In [13] the same result is proved for screw invariant marginally trapped surfaces in $\mathbb{R}_{1}^{4}$.

\section{Fundamental THEOREM}

In the local theory of surfaces in Euclidean space a statement of significant importance is a theorem of Bonnet-type giving the natural conditions under which the surface is determined up to a motion. A theorem of this type was proved for surfaces with flat normal connection by B.-Y. Chen in [1]. In [7] we proved a fundamental theorem of Bonnet-type for surfaces in $\mathbb{R}^{4}$ free of minimal points. In [8] we considered spacelike surfaces in $\mathbb{R}_{1}^{4}$ whose mean curvature vector at any point is a non-zero spacelike vector or timelike vector. Using the geometric moving frame field of Frenet-type on such a surface and the corresponding derivative formulas, we proved a fundamental theorem of Bonnet-type for this class of surfaces, stating that any such a surface is determined up to a motion in $\mathbb{R}_{1}^{4}$ by eight invariant functions satisfying some natural conditions. 
In this section we shall consider marginally trapped surfaces, i.e. spacelike surfaces whose mean curvature vector at any point is a lightlike vector. Let $M^{2}$ be such a surface. We assume that $M^{2}$ is free of flat points, i.e. $(L, M, N) \neq(0,0,0)$, and $M^{2}$ is parameterized by principal lines. Let $\{x, y\}$ be the principal tangent vector fields and $\left\{n_{1}, n_{2}\right\}$ be the pseudo-orthonormal normal frame field, defined in Section 3. Thus we obtain a special frame field $\left\{x, y, n_{1}, n_{2}\right\}$ at each point $p \in M^{2}$, such that $x, y$ are unit spacelike vector fields collinear with the principal directions; $n_{1}, n_{2}$ are lightlike vectors, $\left\langle n_{1}, n_{2}\right\rangle=-1$, and $n_{1}$ is the mean curvature vector field. We call such a frame field a geometric frame field of $M^{2}$.

With respect to this frame field we have the following Frenet-type derivative formulas of $M^{2}$ :

$$
\begin{aligned}
& \nabla_{x}^{\prime} x=\quad \gamma_{1} y+(1+\nu) n_{1} ; \quad \nabla_{x}^{\prime} n_{1}=\quad \mu y+\beta_{1} n_{1} ; \\
& \nabla_{x}^{\prime} y=-\gamma_{1} x \quad+\lambda n_{1}+\mu n_{2} ; \quad \nabla_{y}^{\prime} n_{1}=\mu x \quad+\beta_{2} n_{1} ; \\
& \nabla_{y}^{\prime} x=\quad-\gamma_{2} y+\lambda n_{1}+\mu n_{2} ; \quad \nabla_{x}^{\prime} n_{2}=(1+\nu) x+\lambda y \quad-\beta_{1} n_{2} ; \\
& \nabla_{y}^{\prime} y=\gamma_{2} x \quad+(1-\nu) n_{1} ; \quad \quad \nabla_{y}^{\prime} n_{2}=\lambda x+(1-\nu) y \quad-\beta_{2} n_{2} \text {, }
\end{aligned}
$$

where $\gamma_{1}=-y(\ln \sqrt{E}), \gamma_{2}=-x(\ln \sqrt{G}), \beta_{1}=-\left\langle\nabla_{x}^{\prime} n_{1}, n_{2}\right\rangle, \beta_{2}=-\left\langle\nabla_{y}^{\prime} n_{1}, n_{2}\right\rangle$, and $\mu \neq 0$.

Using that $R^{\prime}\left(x, y, n_{1}\right)=0, R^{\prime}\left(x, y, n_{2}\right)=0$, and $R^{\prime}(x, y, x)=0$, from (5) we get the following integrability conditions:

$$
\begin{aligned}
& x(\mu)-2 \mu \gamma_{2}-\mu \beta_{1}=0 ; \\
& y(\mu)-2 \mu \gamma_{1}-\mu \beta_{2}=0 ; \\
& x\left(\gamma_{2}\right)+y\left(\gamma_{1}\right)-\left(\left(\gamma_{1}\right)^{2}+\left(\gamma_{2}\right)^{2}\right)-2 \lambda \mu=0 ; \\
& x(\lambda)-y(\nu)-2 \lambda \gamma_{2}+2 \nu \gamma_{1}+\lambda \beta_{1}-(1+\nu) \beta_{2}=0 ; \\
& x(\nu)+y(\lambda)-2 \lambda \gamma_{1}-2 \nu \gamma_{2}-(1-\nu) \beta_{1}+\lambda \beta_{2}=0 ; \\
& x\left(\beta_{2}\right)-y\left(\beta_{1}\right)+2 \nu \mu+\gamma_{1} \beta_{1}-\gamma_{2} \beta_{2}=0 .
\end{aligned}
$$

Having in mind that $x=\frac{z_{u}}{\sqrt{E}}, y=\frac{z_{v}}{\sqrt{G}}$, we can rewrite the above equalities as follows:

$$
\begin{aligned}
& 2 \mu \gamma_{2}+\mu \beta_{1}=\frac{1}{\sqrt{E}} \mu_{u} \\
& 2 \mu \gamma_{1}+\mu \beta_{2}=\frac{1}{\sqrt{G}} \mu_{v} \\
& 2 \lambda \mu=\frac{1}{\sqrt{E}}\left(\gamma_{2}\right)_{u}+\frac{1}{\sqrt{G}}\left(\gamma_{1}\right)_{v}-\left(\left(\gamma_{1}\right)^{2}+\left(\gamma_{2}\right)^{2}\right) \\
& 2 \lambda \gamma_{2}-2 \nu \gamma_{1}-\lambda \beta_{1}+(1+\nu) \beta_{2}=\frac{1}{\sqrt{E}} \lambda_{u}-\frac{1}{\sqrt{G}} \nu_{v} \\
& 2 \lambda \gamma_{1}+2 \nu \gamma_{2}+(1-\nu) \beta_{1}-\lambda \beta_{2}=\frac{1}{\sqrt{E}} \nu_{u}+\frac{1}{\sqrt{G}} \lambda_{v} \\
& \gamma_{1} \beta_{1}-\gamma_{2} \beta_{2}+2 \nu \mu=-\frac{1}{\sqrt{E}}\left(\beta_{2}\right)_{u}+\frac{1}{\sqrt{G}}\left(\beta_{1}\right)_{v}
\end{aligned}
$$

The condition $\mu_{u} \mu_{v} \neq 0$ is equivalent to $\left(2 \gamma_{2}+\beta_{1}\right)\left(2 \gamma_{1}+\beta_{2}\right) \neq 0$. So, if $\mu_{u} \mu_{v} \neq 0$, then

$$
\sqrt{E}=\frac{\mu_{u}}{\mu\left(2 \gamma_{2}+\beta_{1}\right)} ; \quad \sqrt{G}=\frac{\mu_{v}}{\mu\left(2 \gamma_{1}+\beta_{2}\right)} .
$$


We shall prove the following Bonnet-type theorem for marginally trapped surfaces in $\mathbb{R}_{1}^{4}$ free of flat points.

Theorem 4.1. Let $\gamma_{1}, \gamma_{2}, \nu, \lambda, \mu, \beta_{1}, \beta_{2}$ be smooth functions, defined in a domain $\mathcal{D}, \mathcal{D} \subset \mathbb{R}^{2}$, and satisfying the conditions

$$
\begin{array}{lc}
\frac{\mu_{u}}{\mu\left(2 \gamma_{2}+\beta_{1}\right)}>0 ; & \frac{\mu_{v}}{\mu\left(2 \gamma_{1}+\beta_{2}\right)}>0 \\
-\gamma_{1} \sqrt{E} \sqrt{G}=(\sqrt{E})_{v} ; & -\gamma_{2} \sqrt{E} \sqrt{G}=(\sqrt{G})_{u} \\
2 \lambda \mu=\frac{1}{\sqrt{E}}\left(\gamma_{2}\right)_{u}+\frac{1}{\sqrt{G}}\left(\gamma_{1}\right)_{v}-\left(\left(\gamma_{1}\right)^{2}+\left(\gamma_{2}\right)^{2}\right) & \\
2 \lambda \gamma_{2}-2 \nu \gamma_{1}-\lambda \beta_{1}+(1+\nu) \beta_{2}=\frac{1}{\sqrt{E}} \lambda_{u}-\frac{1}{\sqrt{G}} \nu_{v} & \\
2 \lambda \gamma_{1}+2 \nu \gamma_{2}+(1-\nu) \beta_{1}-\lambda \beta_{2}=\frac{1}{\sqrt{E}} \nu_{u}+\frac{1}{\sqrt{G}} \lambda_{v} \\
\gamma_{1} \beta_{1}-\gamma_{2} \beta_{2}+2 \nu \mu=-\frac{1}{\sqrt{E}}\left(\beta_{2}\right)_{u}+\frac{1}{\sqrt{G}}\left(\beta_{1}\right)_{v}
\end{array}
$$

where $\sqrt{E}=\frac{\mu_{u}}{\mu\left(2 \gamma_{2}+\beta_{1}\right)}, \sqrt{G}=\frac{\mu_{v}}{\mu\left(2 \gamma_{1}+\beta_{2}\right)}$. Let $\left\{x_{0}, y_{0},\left(n_{1}\right)_{0},\left(n_{2}\right)_{0}\right\}$ be vectors at a point $p_{0} \in \mathbb{R}_{1}^{4}$, such that $x_{0}, y_{0}$ are unit spacelike vectors, $\left\langle x_{0}, y_{0}\right\rangle=0,\left(n_{1}\right)_{0},\left(n_{2}\right)_{0}$ are lightlike vectors, and $\left\langle\left(n_{1}\right)_{0},\left(n_{2}\right)_{0}\right\rangle=-1$. Then there exist a subdomain $\mathcal{D}_{0} \subset \mathcal{D}$ and a unique marginally trapped surface $M^{2}: z=z(u, v),(u, v) \in \mathcal{D}_{0}$ free of flat points, such that $M^{2}$ passes through $p_{0}$, the functions $\gamma_{1}, \gamma_{2}, \nu, \lambda, \mu, \beta_{1}, \beta_{2}$ are the geometric functions of $M^{2}$ and $\left\{x_{0}, y_{0},\left(n_{1}\right)_{0},\left(n_{2}\right)_{0}\right\}$ is the geometric frame of $M^{2}$ at the point $p_{0}$.

Proof: We consider the following system of partial differential equations for the unknown vector functions $x=x(u, v), y=y(u, v), n_{1}=n_{1}(u, v), n_{2}=n_{2}(u, v)$ in $\mathbb{R}_{1}^{4}$ :

$$
\begin{array}{ll}
x_{u}=\sqrt{E}\left(\gamma_{1} y+(1+\nu) n_{1}\right) & x_{v}=\sqrt{G}\left(-\gamma_{2} y+\lambda n_{1}+\mu n_{2}\right) \\
y_{u}=\sqrt{E}\left(-\gamma_{1} x+\lambda n_{1}+\mu n_{2}\right) & y_{v}=\sqrt{G}\left(\gamma_{2} x+(1-\nu) n_{1}\right) \\
\left(n_{1}\right)_{u}=\sqrt{E}\left(\mu y+\beta_{1} n_{1}\right) & \left(n_{1}\right)_{v}=\sqrt{G}\left(\mu x+\beta_{2} n_{1}\right) \\
\left(n_{2}\right)_{u}=\sqrt{E}\left((1+\nu) x+\lambda y-\beta_{1} n_{2}\right) & \left(n_{2}\right)_{v}=\sqrt{G}\left(\lambda x+(1-\nu) y-\beta_{2} n_{2}\right)
\end{array}
$$

We denote

$$
\begin{gathered}
Z=\left(\begin{array}{c}
x \\
y \\
n_{1} \\
n_{2}
\end{array}\right) ; \quad A=\sqrt{E}\left(\begin{array}{cccc}
0 & \gamma_{1} & (1+\nu) & 0 \\
-\gamma_{1} & 0 & \lambda & \mu \\
0 & \mu & \beta_{1} & 0 \\
(1+\nu) & \lambda & 0 & -\beta_{1}
\end{array}\right) ; \\
B=\sqrt{G}\left(\begin{array}{cccc}
0 & -\gamma_{2} & \lambda & \mu \\
\gamma_{2} & 0 & (1-\nu) & 0 \\
\mu & 0 & \beta_{2} & 0 \\
\lambda & (1-\nu) & 0 & -\beta_{2}
\end{array}\right)
\end{gathered}
$$

Then system (7) can be rewritten in the form:

$$
\begin{aligned}
& Z_{u}=A Z, \\
& Z_{v}=B Z .
\end{aligned}
$$


The integrability conditions of (8) are

$$
Z_{u v}=Z_{v u}
$$

i.e.

$$
\frac{\partial a_{i}^{k}}{\partial v}-\frac{\partial b_{i}^{k}}{\partial u}+\sum_{j=1}^{4}\left(a_{i}^{j} b_{j}^{k}-b_{i}^{j} a_{j}^{k}\right)=0, \quad i, k=1, \ldots, 4,
$$

where $a_{i}^{j}$ and $b_{i}^{j}$ are the elements of the matrices $A$ and $B$. Using (6) we obtain that equalities (91) are fulfilled. Hence, there exist a subset $\mathcal{D}_{1} \subset \mathcal{D}$ and unique vector functions $x=x(u, v), y=$ $y(u, v), n_{1}=n_{1}(u, v), n_{2}=n_{2}(u, v),(u, v) \in \mathcal{D}_{1}$, which satisfy system (7) and the conditions

$$
x\left(u_{0}, v_{0}\right)=x_{0}, \quad y\left(u_{0}, v_{0}\right)=y_{0}, \quad n_{1}\left(u_{0}, v_{0}\right)=\left(n_{1}\right)_{0}, \quad n_{2}\left(u_{0}, v_{0}\right)=\left(n_{2}\right)_{0} .
$$

We shall prove that for each $(u, v) \in \mathcal{D}_{1}$ the vectors $x(u, v), y(u, v), n_{1}(u, v), n_{2}(u, v)$ have the following properties: $x(u, v), y(u, v)$ are unit spacelike vectors, $\langle x(u, v), y(u, v)\rangle=0$, $n_{1}(u, v), n_{2}(u, v)$ are lightlike vectors, and $\left\langle n_{1}, n_{2}\right\rangle=-1$. Let us consider the following functions:

$$
\begin{array}{lll}
\varphi_{1}=\langle x, x\rangle-1 ; & \varphi_{5}=\langle x, y\rangle ; & \varphi_{8}=\left\langle y, n_{1}\right\rangle ; \\
\varphi_{2}=\langle y, y\rangle-1 ; & \varphi_{6}=\left\langle x, n_{1}\right\rangle ; & \varphi_{9}=\left\langle y, n_{2}\right\rangle ; \\
\varphi_{3}=\left\langle n_{1}, n_{1}\right\rangle ; & \varphi_{7}=\left\langle x, n_{2}\right\rangle ; & \varphi_{10}=\left\langle n_{1}, n_{2}\right\rangle+1 ; \\
\varphi_{4}=\left\langle n_{2}, n_{2}\right\rangle ; & &
\end{array}
$$

defined for each $(u, v) \in \mathcal{D}_{1}$. Using that $x(u, v), y(u, v), n_{1}(u, v), n_{2}(u, v)$ satisfy (77), we obtain the system

$$
\begin{aligned}
& \frac{\partial \varphi_{i}}{\partial u}=\alpha_{i}^{j} \varphi_{j}, \\
& \frac{\partial \varphi_{i}}{\partial v}=\beta_{i}^{j} \varphi_{j} ; \quad i=1, \ldots, 10,
\end{aligned}
$$

where $\alpha_{i}^{j}, \beta_{i}^{j}, i, j=1, \ldots, 10$ are functions of $(u, v) \in \mathcal{D}_{1}$. System (10) is a linear system of partial differential equations for the functions $\varphi_{i}(u, v), i=1, \ldots, 10,(u, v) \in \mathcal{D}_{1}$, satisfying $\varphi_{i}\left(u_{0}, v_{0}\right)=0, i=1, \ldots, 10$. Hence, $\varphi_{i}(u, v)=0, i=1, \ldots, 10$ for each $(u, v) \in \mathcal{D}_{1}$. Consequently, the quadruple $\left\{x(u, v), y(u, v), n_{1}(u, v), n_{2}(u, v)\right\}$ has the properties mentioned above.

Now, let us consider the system

$$
\begin{aligned}
& z_{u}=\sqrt{E} x \\
& z_{v}=\sqrt{G} y
\end{aligned}
$$

of partial differential equations for the vector function $z(u, v)$. Using (6) and (7) we get that the integrability conditions $z_{u v}=z_{v u}$ of system (11) are fulfilled. Hence, there exist a subset $\mathcal{D}_{0} \subset \mathcal{D}_{1}$ and a unique vector function $z=z(u, v)$, defined for $(u, v) \in \mathcal{D}_{0}$ and satisfying $z\left(u_{0}, v_{0}\right)=p_{0}$.

Consequently, the surface $M^{2}: z=z(u, v),(u, v) \in \mathcal{D}_{0}$ satisfies the assertion of the theorem.

Marginally trapped surfaces for which $\beta_{1}=\beta_{2}=0$ have parallel mean curvature vector field, i.e. $D H=0$ holds identically. The class of marginally trapped surfaces with parallel mean curvature vector field, was classified by B.-Y. Chen and J. Van Der Veken in [4]. 


\section{MERIDIAN SURFACES IN $\mathbb{R}_{1}^{4}$}

In [7] we constructed a family of surfaces lying on a standard rotational hypersurface in the four-dimensional Euclidean space $\mathbb{R}^{4}$. These surfaces are one-parameter systems of meridians of the rotational hypersurface, that is why we called them meridian surfaces. We described the meridian surfaces with constant Gauss curvature, with constant mean curvature, and with constant invariant $k$.

In this section we shall use the same idea to construct a special family of two-dimensional spacelike surfaces lying on rotational hypersurfaces in $\mathbb{R}_{1}^{4}$. We shall consider the standard rotational hypersurface with timelike axis and the rotational hypersurface with spacelike axis.

Let $\left\{e_{1}, e_{2}, e_{3}, e_{4}\right\}$ be the standard orthonormal frame in $\mathbb{R}_{1}^{4}$, i.e. $e_{1}^{2}=e_{2}^{2}=e_{3}^{2}=1, e_{4}^{2}=$ -1 . First we consider the standard rotational hypersurface with timelike axis. Let $S^{2}(1)$ be a 2-dimensional sphere in the Euclidean space $\mathbb{R}^{3}=\operatorname{span}\left\{e_{1}, e_{2}, e_{3}\right\}$, centered at the origin $O$. Let $f=f(u), g=g(u)$ be smooth functions, defined in an interval $I \subset \mathbb{R}$, such that $\dot{f}^{2}(u)-\dot{g}^{2}(u)>0, u \in I$, where $\dot{f}(u)$ denotes the derivative $\frac{d f(u)}{d u}$ and $\dot{g}(u)=\frac{d g(u)}{d u}$. We assume that $f(u)>0, u \in I$. The standard rotational hypersurface $\mathcal{M}^{\prime}$ in $\mathbb{R}_{1}^{4}$, obtained by the rotation of the meridian curve $m: u \rightarrow(f(u), g(u))$ about the $O e_{4}$-axis, is parameterized as follows:

$$
\mathcal{M}^{\prime}: Z\left(u, w^{1}, w^{2}\right)=f(u) l\left(w^{1}, w^{2}\right)+g(u) e_{4},
$$

where $l\left(w^{1}, w^{2}\right)$ is the unit position vector of $S^{2}(1)$ in $\mathbb{R}^{3}$. The hypersurface $\mathcal{M}^{\prime}$ is a rotational hypersurface in $\mathbb{R}_{1}^{4}$ with timelike axis.

We consider a smooth curve $c: l=l(v)=l\left(w^{1}(v), w^{2}(v)\right), v \in J, J \subset \mathbb{R}$ on $S^{2}(1)$, parameterized by the arc-length, i.e. $\left\langle l^{\prime}(v), l^{\prime}(v)\right\rangle=1$. Let $t(v)=l^{\prime}(v)$ be the tangent vector field of $c$. Since $\langle t(v), t(v)\rangle=1,\langle l(v), l(v)\rangle=1$, and $\langle t(v), l(v)\rangle=0$, there exists a unique (up to a sign) vector field $n(v)$ in $\mathbb{R}^{3}$, such that $\{l(v), t(v), n(v)\}$ is an orthonormal frame field. With respect to this orthonormal frame field we have the following Frenet formulas of $c$ on $S^{2}(1)$ :

$$
\begin{aligned}
& l^{\prime}=t ; \\
& t^{\prime}=\kappa n-l ; \\
& n^{\prime}=-\kappa t,
\end{aligned}
$$

where $\kappa(v)=\left\langle t^{\prime}(v), n(v)\right\rangle$ is the spherical curvature of $c$.

Now we construct a surface $\mathcal{M}_{m}^{\prime}$ in $\mathbb{R}_{1}^{4}$ in the following way:

$$
\mathcal{M}_{m}^{\prime}: z(u, v)=f(u) l(v)+g(u) e_{4}, \quad u \in I, v \in J
$$

The surface $\mathcal{M}_{m}^{\prime}$ lies on the rotational hypersurface $\mathcal{M}^{\prime}$ in $\mathbb{R}_{1}^{4}$. Since $\mathcal{M}_{m}^{\prime}$ is a one-parameter system of meridians of $\mathcal{M}^{\prime}$, we call $\mathcal{M}_{m}^{\prime}$ a meridian surface on $\mathcal{M}^{\prime}$.

The tangent space of $\mathcal{M}_{m}^{\prime}$ is spanned by the vector fields:

$$
\begin{aligned}
& z_{u}=\dot{f} l+\dot{g} e_{4} ; \\
& z_{v}=f t
\end{aligned}
$$

so, the coefficients of the first fundamental form of $\mathcal{M}_{m}^{\prime}$ are

$$
E=\dot{f}^{2}(u)-\dot{g}^{2}(u)>0 ; \quad F=0 ; \quad G=f^{2}(u)>0 .
$$

Hence, the first fundamental form is positive definite, i.e. $\mathcal{M}_{m}^{\prime}$ is a spacelike surface is $\mathbb{R}_{1}^{4}$. 
Let us denote $x=\frac{z_{u}}{\sqrt{\dot{f}^{2}-\dot{g}^{2}}}, y=\frac{z_{v}}{f}$. Then we have the following orthonormal tangent frame field of $\mathcal{M}_{m}^{\prime}$ :

$$
x=\frac{\dot{f}(u)}{\sqrt{\dot{f}^{2}(u)-\dot{g}^{2}(u)}} l(v)+\frac{\dot{g}(u)}{\sqrt{\dot{f}^{2}(u)-\dot{g}^{2}(u)}} e_{4} ; \quad y=t(v) .
$$

We consider the orthonormal normal frame field, defined by:

$$
n_{1}=n(v) ; \quad n_{2}=\frac{\dot{g}(u)}{\sqrt{\dot{f}^{2}(u)-\dot{g}^{2}(u)}} l(v)+\frac{\dot{f}(u)}{\sqrt{\dot{f}^{2}(u)-\dot{g}^{2}(u)}} e_{4} .
$$

Thus we obtain a frame field $\left\{x, y, n_{1}, n_{2}\right\}$ of $\mathcal{M}_{m}^{\prime}$, such that $\left\langle n_{1}, n_{1}\right\rangle=1,\left\langle n_{2}, n_{2}\right\rangle=-1$, $\left\langle n_{1}, n_{2}\right\rangle=0$.

Taking into account (12), we calculate the second partial derivatives of $z(u, v)$ :

$$
\begin{aligned}
& z_{u u}=\ddot{f} l+\ddot{g} e_{4} ; \\
& z_{u v}=\dot{f} t ; \\
& z_{v v}=f \kappa n-f l .
\end{aligned}
$$

Then we get

$$
\begin{aligned}
& c_{11}^{1}=\left\langle z_{u u}, n_{1}\right\rangle=0 ; \quad c_{12}^{1}=\left\langle z_{u v}, n_{1}\right\rangle=0 ; \quad c_{22}^{1}=\left\langle z_{v v}, n_{1}\right\rangle=f \kappa ; \\
& c_{11}^{2}=\left\langle z_{u u}, n_{2}\right\rangle=\frac{\ddot{f} \dot{g}-\ddot{g} \dot{f}}{\sqrt{\dot{f}^{2}-\dot{g}^{2}}} ; \quad c_{12}^{2}=\left\langle z_{u v}, n_{2}\right\rangle=0 ; \quad c_{22}^{2}=\left\langle z_{v v}, n_{2}\right\rangle=-\frac{f \dot{g}}{\sqrt{\dot{f}^{2}-\dot{g}^{2}}} .
\end{aligned}
$$

Hence,

$$
\begin{aligned}
& \sigma(x, x)=\quad \frac{\dot{f} \ddot{g}-\dot{g} \ddot{f}}{\left(\dot{f}^{2}-\dot{g}^{2}\right)^{\frac{3}{2}}} n_{2} \\
& \sigma(x, y)=0 ; \\
& \sigma(y, y)=\frac{\kappa}{f} n_{1}+\frac{\dot{g}}{f \sqrt{\dot{f}^{2}-\dot{g}^{2}}} n_{2} .
\end{aligned}
$$

Let us denote by $\kappa_{m}$ the curvature of the meridian curve $m$, i.e. $\kappa_{m}(u)=\frac{\dot{f}(u) \ddot{g}(u)-\dot{g}(u) \ddot{f}(u)}{\left(\dot{f}^{2}(u)-\dot{g}^{2}(u)\right)^{\frac{3}{2}}}$. Taking into account (14), we find the invariants $k, \varkappa$, and the Gauss curvature $K$ of $\mathcal{M}_{m}^{\prime}$ :

$$
k=-\frac{\kappa_{m}^{2}(u) \kappa^{2}(v)}{f^{2}(u)} ; \quad \varkappa=0 ; \quad K=-\frac{\kappa_{m}(u) \dot{g}(u)}{f(u) \sqrt{\dot{f}^{2}(u)-\dot{g}^{2}(u)}} .
$$

The equality $\varkappa=0$ implies that $\mathcal{M}_{m}^{\prime}$ is a surface with flat normal connection.

The mean curvature vector field $H$ is given by

$$
H=\frac{\kappa}{2 f} n_{1}+\frac{\kappa_{m} f \sqrt{\dot{f}^{2}-\dot{g}^{2}}+\dot{g}}{2 f \sqrt{\dot{f}^{2}-\dot{g}^{2}}} n_{2} .
$$


Equality (15) implies that $\mathcal{M}_{m}^{\prime}$ is a minimal surface (the mean curvature vector field $H$ is zero) if and only if

$$
\kappa=0 ; \quad \kappa_{m} f \sqrt{\dot{f}^{2}-\dot{g}^{2}}+\dot{g}=0 .
$$

We shall exclude this case and consider the case when $\mathcal{M}_{m}^{\prime}$ is marginally trapped, i.e. $H \neq 0$ and $\langle H, H\rangle=0$. Using (15) we obtain the following result.

Proposition 5.1. The meridian surface $\mathcal{M}_{m}^{\prime}$, defined by (13), is marginally trapped if and only if

$$
\kappa^{2}=\frac{\left(\kappa_{m} f \sqrt{\dot{f}^{2}-\dot{g}^{2}}+\dot{g}\right)^{2}}{\dot{f}^{2}-\dot{g}^{2}}, \quad \kappa \neq 0 .
$$

In a similar way we shall consider meridian surfaces lying on the standard rotational hypersurface in $\mathbb{R}_{1}^{4}$ with spacelike axis. Let $S_{1}^{2}(1)$ be the timelike sphere in the Minkowski space $\mathbb{R}_{1}^{3}=\operatorname{span}\left\{e_{2}, e_{3}, e_{4}\right\}$, i.e. $S_{1}^{2}(1)=\left\{V \in \mathbb{R}_{1}^{3}:\langle V, V\rangle=1\right\} . S_{1}^{2}(1)$ is a timelike surface in $\mathbb{R}_{1}^{3}$ known as the de Sitter space. Let $f=f(u), g=g(u)$ be smooth functions, defined in an interval $I \subset \mathbb{R}$, such that $\dot{f}^{2}(u)+\dot{g}^{2}(u)>0, f(u)>0, u \in I$. We denote by $l\left(w^{1}, w^{2}\right)$ the unit position vector of $S_{1}^{2}(1)$ in $\mathbb{R}_{1}^{3}$ and consider the rotational hypersurface $\mathcal{M}^{\prime \prime}$ in $\mathbb{R}_{1}^{4}$, obtained by the rotation of the meridian curve $m: u \rightarrow(f(u), g(u))$ about the $O e_{1}$-axis. It is parameterized as follows:

$$
\mathcal{M}^{\prime \prime}: Z\left(u, w^{1}, w^{2}\right)=f(u) l\left(w^{1}, w^{2}\right)+g(u) e_{1} .
$$

The hypersurface $\mathcal{M}^{\prime \prime}$ is a rotational hypersurface in $\mathbb{R}_{1}^{4}$ with spacelike axis.

Now we consider a smooth spacelike curve $c: l=l(v)=l\left(w^{1}(v), w^{2}(v)\right), v \in J, J \subset \mathbb{R}$ on $S_{1}^{2}(1)$, parameterized by the arc-length, i.e. $\left\langle l^{\prime}(v), l^{\prime}(v)\right\rangle=1$, and denote by $t(v)=l^{\prime}(v)$ the tangent vector field of $c$. Since $\langle t(v), t(v)\rangle=1,\langle l(v), l(v)\rangle=1$, and $\langle t(v), l(v)\rangle=0$, there exists a unique (up to a sign) timelike vector field $n(v)$ in $\mathbb{R}_{1}^{3}$, such that $\langle n(v), n(v)\rangle=$ $-1,\langle n(v), t(v)\rangle=0,\langle n(v), l(v)\rangle=0$. In such a way we obtain an orthonormal frame field $\{l(v), t(v), n(v)\}$ in $\mathbb{R}_{1}^{3}$. With respect to this frame field we have the following decompositions of the vector fields $l^{\prime}(v), t^{\prime}(v), n^{\prime}(v)$ :

$$
\begin{aligned}
& l^{\prime}=t ; \\
& t^{\prime}=-\kappa n-l ; \\
& n^{\prime}=-\kappa t,
\end{aligned}
$$

which can be considered as Frenet formulas of $c$ on $S_{1}^{2}(1)$. The function $\kappa(v)=\left\langle t^{\prime}(v), n(v)\right\rangle$ is the spherical curvature of $c$ on $S_{1}^{2}(1)$.

We construct a surface $\mathcal{M}_{m}^{\prime \prime}$ in $\mathbb{R}_{1}^{4}$ in the following way:

$$
\mathcal{M}_{m}^{\prime \prime}: z(u, v)=f(u) l(v)+g(u) e_{1}, \quad u \in I, v \in J
$$

The surface $\mathcal{M}_{m}^{\prime \prime}$, defined by (16), lies on the rotational hypersurface $\mathcal{M}^{\prime \prime}$ in $\mathbb{R}_{1}^{4}$. Since $\mathcal{M}_{m}^{\prime \prime}$ is a one-parameter system of meridians of $\mathcal{M}^{\prime \prime}$, we call $\mathcal{M}_{m}^{\prime \prime}$ a meridian surface on $\mathcal{M}^{\prime \prime}$.

The meridian surface $\mathcal{M}_{m}^{\prime \prime}$ is a spacelike surface is $\mathbb{R}_{1}^{4}$ with tangent vector fields

$$
\begin{aligned}
& z_{u}=\dot{f} l+\dot{g} e_{1} ; \\
& z_{v}=f t,
\end{aligned}
$$

and coefficients of the first fundamental form given by

$$
E=\dot{f}^{2}(u)+\dot{g}^{2}(u)>0 ; \quad F=0 ; \quad G=f^{2}(u)>0 .
$$


We consider the orthonormal tangent frame field $x=\frac{z_{u}}{\sqrt{\dot{f}^{2}+\dot{g}^{2}}}, y=\frac{z_{v}}{f}$, i.e.

$$
x=\frac{\dot{f}(u)}{\sqrt{\dot{f}^{2}(u)+\dot{g}^{2}(u)}} l(v)+\frac{\dot{g}(u)}{\sqrt{\dot{f}^{2}(u)+\dot{g}^{2}(u)}} e_{1} ; \quad y=t(v),
$$

and the orthonormal normal frame field, defined by:

$$
n_{1}=\frac{\dot{g}(u)}{\sqrt{\dot{f}^{2}(u)+\dot{g}^{2}(u)}} l(v)-\frac{\dot{f}(u)}{\sqrt{\dot{f}^{2}(u)+\dot{g}^{2}(u)}} e_{1} ; \quad n_{2}=n(v) .
$$

Thus we obtain a frame field $\left\{x, y, n_{1}, n_{2}\right\}$ of $\mathcal{M}_{m}^{\prime \prime}$, such that $\left\langle n_{1}, n_{1}\right\rangle=1,\left\langle n_{2}, n_{2}\right\rangle=-1$, $\left\langle n_{1}, n_{2}\right\rangle=0$.

In the same way as in the previous example, we obtain the formulas

$$
\begin{aligned}
& \sigma(x, x)=\frac{\dot{g} \ddot{f}-\dot{f} \ddot{g}}{\left(\dot{f}^{2}+\dot{g}^{2}\right)^{\frac{3}{2}}} n_{1} ; \\
& \sigma(x, y)=0 ; \\
& \sigma(y, y)=-\frac{\dot{g}}{f \sqrt{\dot{f}^{2}+\dot{g}^{2}}} n_{1}-\frac{\kappa}{f} n_{2} .
\end{aligned}
$$

Now, the curvature $\kappa_{m}$ of the meridian curve $m$ is given by $\kappa_{m}(u)=\frac{\dot{f}(u) \ddot{g}(u)-\dot{g}(u) \ddot{f}(u)}{\left(\dot{f}^{2}(u)+\dot{g}^{2}(u)\right)^{\frac{3}{2}}}$. The invariants $k, \varkappa$, and the Gauss curvature $K$ of $\mathcal{M}_{m}^{\prime \prime}$ are expressed as follows:

$$
k=-\frac{\kappa_{m}^{2}(u) \kappa^{2}(v)}{f^{2}(u)} ; \quad \varkappa=0 ; \quad K=\frac{\kappa_{m}(u) \dot{g}(u)}{f(u) \sqrt{\dot{f}^{2}(u)+\dot{g}^{2}(u)}} .
$$

The equality $\varkappa=0$ implies that $\mathcal{M}_{m}^{\prime \prime}$ is a surface with flat normal connection.

The mean curvature vector field $H$ is given by

$$
H=-\frac{\kappa_{m} f \sqrt{\dot{f}^{2}+\dot{g}^{2}}+\dot{g}}{2 f \sqrt{\dot{f}^{2}+\dot{g}^{2}}} n_{1}-\frac{\kappa}{2 f} n_{2} .
$$
if

Obviously, $\mathcal{M}_{m}^{\prime \prime}$ is a minimal surface (the mean curvature vector field $H$ is zero) if and only

$$
\kappa=0 ; \quad \kappa_{m} f \sqrt{\dot{f}^{2}+\dot{g}^{2}}+\dot{g}=0 .
$$

We consider the case when $\mathcal{M}_{m}^{\prime \prime}$ is marginally trapped, i.e. $H \neq 0$ and $\langle H, H\rangle=0$. Equality (17) implies the following result.

Proposition 5.2. The meridian surface $\mathcal{M}_{m}^{\prime \prime}$, defined by (16), is marginally trapped if and only if

$$
\kappa^{2}=\frac{\left(\kappa_{m} f \sqrt{\dot{f}^{2}+\dot{g}^{2}}+\dot{g}\right)^{2}}{\dot{f}^{2}+\dot{g}^{2}}, \quad \kappa \neq 0
$$


At the end of this section we shall find all marginally trapped meridian surfaces lying on the rotational hypersurfaces $\mathcal{M}^{\prime}$ or $\mathcal{M}^{\prime \prime}$.

Let $\mathcal{M}_{m}^{\prime}$ be a marginally trapped meridian surface lying on the rotational hypersurface $\mathcal{M}^{\prime}$. According to Proposition 5.1 the following equality holds:

$$
\kappa^{2}(v)=\frac{\left(\kappa_{m}(u) f(u) \sqrt{\dot{f}^{2}(u)-\dot{g}^{2}(u)}+\dot{g}(u)\right)^{2}}{\dot{f}^{2}(u)-\dot{g}^{2}(u)},
$$

which imply

$$
\begin{aligned}
& \kappa(v)=a=\text { const }, \quad a \neq 0 \\
& \frac{\kappa_{m}(u) f(u) \sqrt{\dot{f}^{2}(u)-\dot{g}^{2}(u)}+\dot{g}(u)}{\sqrt{\dot{f}^{2}(u)-\dot{g}^{2}(u)}}= \pm a .
\end{aligned}
$$

Without loss of generality we assume that the meridian curve $m$ is given by

$$
f=u ; \quad g=g(u) .
$$

Then $\dot{f}(u)=1 ; \ddot{f}(u)=0 ; \kappa_{m}(u)=\frac{\ddot{g}}{\left(1-\dot{g}^{2}\right)^{\frac{3}{2}}}$. Hence, equation (18) takes the form:

$$
u \ddot{g}+\dot{g}-(\dot{g})^{3}= \pm a\left(1-\dot{g}^{2}\right)^{\frac{3}{2}} .
$$

The meridian curves of all marginally trapped meridian surfaces lying on the rotational hypersurface $\mathcal{M}^{\prime}$ are determined by the solutions of differential equation (19). After the change $\frac{1}{1-\dot{g}^{2}(u)}=h^{2}(u)+1$ the above equation is transformed into

$$
u \dot{h}(u)+h(u)= \pm a \varepsilon, \quad \varepsilon= \pm 1 .
$$

The general solution of equation (20) is given by

$$
h(u)=\frac{ \pm a \varepsilon u+c_{1}}{u}, \quad c_{1}=\text { const. }
$$

We set $c=\varepsilon c_{1}$ and get

$$
\dot{g}(u)=\frac{ \pm a u+c}{\sqrt{( \pm a u+c)^{2}+u^{2}}} .
$$

Integrating (21) we obtain that all solutions of differential equation (19) are given by the formula

$g(u)=\frac{ \pm a}{a^{2}+1} \sqrt{( \pm a u+c)^{2}+u^{2}}+\frac{c}{\left(a^{2}+1\right)^{\frac{3}{2}}} \ln \left(\sqrt{a^{2}+1} u \pm \frac{a c}{\sqrt{a^{2}+1}}+\sqrt{( \pm a u+c)^{2}+u^{2}}\right)+b$,

where $b$ and $c$ are constants, $c \neq 0$.

Thus we obtain the next result.

Theorem 5.3. The meridian surface $\mathcal{M}_{m}^{\prime}$ is marginally trapped if and only if the curve $c$ on $S^{2}(1)$ has constant spherical curvature $\kappa=a, a \neq 0$, and the meridian $m$ is defined by (22). 
Let $\mathcal{M}_{m}^{\prime}$ be a marginally trapped meridian surface. Then the meridian curve $m$ is determined by $f=u, g=g(u)$, where the function $g(u)$ is defined by (22). Hence, the curvature of $m$ is $\kappa_{m}=-\frac{c}{u^{2}}$. Formulas (14) take the form

$$
\begin{aligned}
& \sigma(x, x)=\quad-\frac{c}{u^{2}} n_{2} ; \\
& \sigma(x, y)=0 ; \\
& \sigma(y, y)=\frac{a}{u} n_{1}+\frac{ \pm a u+c}{u^{2}} n_{2},
\end{aligned}
$$

which imply that the invariants $k, \varkappa$ and $K$ of $\mathcal{M}_{m}^{\prime}$ are expressed as

$$
k=-\frac{a^{2} c^{2}}{u^{6}} ; \quad \varkappa=0 ; \quad K=\frac{c( \pm a u+c)}{u^{4}} .
$$

The mean curvature vector field of $\mathcal{M}_{m}^{\prime}$ is $H=\frac{a}{2 u}\left(n_{1} \pm n_{2}\right)$.

Note that $\left\{x, y, n_{1}, n_{2}\right\}$ is not the geometric frame field of $\mathcal{M}_{m}^{\prime}$ defined in Section 4 . The geometric frame field $\left\{X, Y, N_{1}, N_{2}\right\}$ of $\mathcal{M}_{m}^{\prime}$ is determined by

$$
\begin{aligned}
X & =\frac{1}{\sqrt{2}}(x+y) ; & N_{1} & =\frac{a}{2 u}\left(n_{1} \pm n_{2}\right) ; \\
Y & =\frac{1}{\sqrt{2}}(x-y) ; & N_{2} & =\frac{u}{a}\left(-n_{1} \pm n_{2}\right) .
\end{aligned}
$$

Hence, using (23) we obtain the formulas corresponding to the geometric frame field:

$$
\begin{aligned}
& \sigma(X, X)=N_{1} ; \\
& \sigma(X, Y)=\frac{-a u \mp c}{a u} N_{1} \mp \frac{a c}{2 u^{3}} N_{2} ; \\
& \sigma(Y, Y)=N_{1} .
\end{aligned}
$$

Thus we obtain the invariants $\nu, \lambda, \mu$ in the Frenet-type derivative formulas of $\mathcal{M}_{m}^{\prime}$ :

$$
\nu=0 ; \quad \lambda=\frac{-a u \mp c}{a u} ; \quad \mu=\mp \frac{a c}{2 u^{3}} .
$$

The invariants $\gamma_{1}, \gamma_{2}, \beta_{1}, \beta_{2}$ are defined by

$$
\gamma_{1}=\left\langle\nabla_{X}^{\prime} X, Y\right\rangle ; \quad \gamma_{2}=\left\langle\nabla_{Y}^{\prime} Y, X\right\rangle ; \quad \beta_{1}=-\left\langle\nabla_{X}^{\prime} N_{1}, N_{2}\right\rangle ; \quad \beta_{2}=-\left\langle\nabla_{Y}^{\prime} N_{1}, N_{2}\right\rangle .
$$

Calculating these scalar products we get

$$
\gamma_{1}=\gamma_{2}=-\frac{\sqrt{( \pm a u+c)^{2}+u^{2}}}{\sqrt{2} u^{2}} ; \quad \beta_{1}=\beta_{2}=\frac{\sqrt{( \pm a u+c)^{2}+u^{2}}}{\sqrt{2} u^{4}}\left(c( \pm a u+c)-u^{2}\right) .
$$

Hence, the marginally trapped meridian surface $\mathcal{M}_{m}^{\prime}$ has non-parallel mean curvature vector field, since $\beta_{1}$ and $\beta_{2}$ are non-zero functions.

In a similar way we find all marginally trapped meridian surfaces lying on the rotational hypersurface $\mathcal{M}^{\prime \prime}$. 
Let $\mathcal{M}_{m}^{\prime \prime}$ be a marginally trapped meridian surface lying on $\mathcal{M}^{\prime \prime}$. Applying Proposition 5.2 we obtain the following conditions on the meridian curve $m$ and the curve $c$ lying on $S_{1}^{2}(1)$ :

$$
\begin{aligned}
& \kappa(v)=a=\text { const, } \quad a \neq 0 ; \\
& \frac{\kappa_{m}(u) f(u) \sqrt{\dot{f}^{2}(u)+\dot{g}^{2}(u)}+\dot{g}(u)}{\sqrt{\dot{f}^{2}(u)+\dot{g}^{2}(u)}}= \pm a .
\end{aligned}
$$

If the meridian curve $m$ is given by $f=u ; g=g(u)$, then we get that the function $g(u)$ is a solution of the following differential equation:

$$
u \ddot{g}+\dot{g}+(\dot{g})^{3}= \pm a\left(1+\dot{g}^{2}\right)^{\frac{3}{2}} .
$$

All solutions of this equation are given by the formula

$g(u)=\frac{ \pm a}{1-a^{2}} \sqrt{u^{2}-( \pm a u+c)^{2}}+\frac{c}{\left(1-a^{2}\right)^{\frac{3}{2}}} \ln \left(\sqrt{1-a^{2}} u \mp \frac{a c}{\sqrt{1-a^{2}}}+\sqrt{u^{2}-( \pm a u+c)^{2}}\right)+b$,

where $b$ and $c$ are constants, $c \neq 0$.

Thus we obtain the following result.

Theorem 5.4. The meridian surface $\mathcal{M}_{m}^{\prime \prime}$ is marginally trapped if and only if the curve $c$ on $S_{1}^{2}(1)$ has constant spherical curvature $\kappa=a, a \neq 0$, and the meridian $m$ is defined by (24).

The invariants of the marginally trapped meridian surface $\mathcal{M}_{m}^{\prime \prime}$ are expressed in a similar way as the invariants of the marginally trapped meridian surface $\mathcal{M}_{m}^{\prime}$. The marginally trapped meridian surface $\mathcal{M}_{m}^{\prime \prime}$ has non-parallel mean curvature vector field, since the invarianst $\beta_{1}$ and $\beta_{2}$ are non-zero.

Acknowledgements: The second author is partially supported by "L. Karavelov" Civil Engineering Higher School, Sofia, Bulgaria under Contract No 10/2010.

\section{REFERENCES}

[1] Chen B.-Y., Geometry of submanifolds. Marcel Dekker, Inc., New York, 1973.

[2] Chen B.-Y., Van der Veken J., Marginally trapped surfaces in Lorenzian space with positive relative nullity. Class. Quantum Grav. 24 (2007), 551-563.

[3] Chen B.-Y., Van der Veken J., Spacial and Lorenzian surfaces in Robertson-Walker space-times. J. Math. Phys. 48 (2007) 073509, 12 pp.

[4] Chen B.-Y., Van der Veken J., Classification of marginally trapped surfaces with parallel mean curvature vector in Lorenzian space forms. Houston J. Math. 36 (2010), 421-449.

[5] Ganchev G., Milousheva V., On the theory of surfaces in the four-dimensional Euclidean space, Kodai Math. J., 2008, 31, 183-198.

[6] Ganchev G., Milousheva V., Invariants of lines on surfaces in $\mathbb{R}^{4}$, C. R. Acad. Bulg. Sci., 2010, 63, (6), 835-842.

[7] Ganchev G., Milousheva V., Invariants and Bonnet-type theorem for surfaces in $\mathbb{R}^{4}$, Cent. Eur. J. Math., 2010, 8 (6), 993-1008.

[8] Ganchev G., Milousheva V., An invariant theory of spacelike surfaces in the four-dimensional Minkowski space, Mediterr. J. Math., DOI: 10.1007/s00009-010-0108-2.

[9] Gheysens L., P. Verheyen and L. Verstraelen, Sur les surfaces $\mathcal{A}$ ou les surfaces de Chen. C. R. Acad. Sci. Paris, Sér. I 292 (1981), 913-916.

[10] Gheysens L., P. Verheyen and L. Verstraelen, Characterization and examples of Chen submanifolds. J. Geom. 20 (1983), 47-62.

[11] Haesen S., Ortega M., Boost invariant marginally trapped surfaces in Minkowski 4-space. Class. Quantum Grav. 24 (2007), 5441-5452. 
[12] Haesen S., Ortega M., Marginally trapped surfaces in Minkowksi 4-space invariant under a rotational subgroup of the Lorenz group. Gen. Relativ. Grav. 41 (2009), 1819-1834.

[13] Haesen S., Ortega M., Screw invariant marginally trapped surfaces in Minkowski 4-space. J. Math. Anal. Appl. 355 (2009), 639-648.

[14] Penrose R. Gravitational collapse and space-time singularities. Phys. Rev. Lett., 14 (1965), 57-59.

Bulgarian Academy of Sciences, Institute of Mathematics and Informatics, Acad. G. Bonchev Str. Bl. 8, 1113 Sofia, Bulgaria

E-mail address: ganchev@math.bas.bg

Bulgarian Academy of Sciences, Institute of Mathematics and Informatics, Acad. G. Bonchev Str. Bl. 8, 1113, Sofia, Bulgaria; "L. Karavelov" Civil Engineering Higher School, 175 Suhodolska Str., 1373 Sofia, Bulgaria

E-mail address: vmil@math.bas.bg 